\title{
AIDS, IV drug use and mycobacterial disease: the Dublin experience
}

\author{
E. Healy*†, P. Kelly*, F. M. Mulcahy $\dagger$ And L. Clancy* ${ }^{\dagger}+$ \\ *Peamount Hospital, Newcastle, Co. Dublin and† St. James'Hospital, Dublin 8, Ireland
}

In the period 1980-1985, we treated 1641 patients for tuberculosis of whom two were known to be intravenous drug users (IVDU) and none had HIV infection. Of the next 1000 patients treated for tuberculosis (January 1986-December 1989), six were HIV-negative intravenous drug users (IVDU), 18 patients were HIV-positive (12 IVDU; six homosexual/bisexual). Statistical analysis $\left(X^{2}\right)$ showed a numerically small but statistically significant $\left(P<0.00001\right.$, d.f. $\left.=1, X^{2}=20-38\right)$ increase in intravenous drug users with a diagnosis of tuberculosis. The HIV-positive patients who completed treatment responded well to antituberculous drugs.

The importance of tuberculosis in the context of HIV infection is that it is preventable, treatable and is the only Bacterial infection to which HIV subjects are prone which can be readily transmitted to a non-HIV infected subject.

\section{Introduction}

Tuberculosis (TB) is well recognized as one of the most common bacterial infections in patients infected with Human Immunodeficiency Virus (HIV). While the lifetime risk of active disease following primary infection is said to be the order of $10 \%$ in an immunocompetent subject, the risk of active disease is believed to be up to $30 \%$ in a HIV-positive subject (1). It is possible that tuberculosis, even if successfully treated, may accelerate the progression of HIV infection to AIDS $(2,3)$. Tuberculosis in otherwise normal individuals has a number of immunosuppressive effects including increased production of acute phase reactants, some of which may suppress certain lymphocytic functions (4), and even induction of CD4 lymphopenia (5). Immunological mediators produced during infection may augment the replication of HIV virus in lymphocytes and monocytes.

A steady increase in the incidence of tuberculosis in the United States of America has been attributed to the AIDS epidemic (1). The question is whether tuberculosis among HIV-positive subjects is due to recent infection and early progression of disease or due to reactivation of previous latent infection. In their study in New York, Selwyn et al. (6) suggested that in HIV infected persons, tuberculosis most often resulted from reactivation of latent disease. It is possible, however, that in HIV-positive subjects, because of declining immunocompetence, that tuberculosis may

Received 15 April 1991 and accepted in revised form 31 January 1992.

To whom correspondence should be addressed. become rapidly progressive to active disease following recent infection.

We have previously noted the occurrence of tuberculosis in patients infected with HIV in the Republic of Ireland (7). We have also reported the incidence of disease due to mycobacteria other than tuberculosis (MOTT) as eight per 1000 positive myco-bacterial cultures (8). In a retrospective study covering the period January 1980-December 1985, only two out of 1641 patients treated for tuberculosis were known intravenous drug users (IVDU). Their HIV status is unknown.

Tuberculosis in HIV-positive subjects may be difficult to diagnose as the typical radiological appearances may not occur. Indeed many of the features are more consistent with primary infection (9-11). The tuberculosis is not confined to the apices, cavitation may be absent, there may be widespread pulmonary involvement and frequently there is hilar or medias-tinal gland involvement. There is also frequently extrapulmonary disease $(9,10,12)$.

We treat all patients with tuberculosis, including patients who are HIV-positive with standard anti-tuberculous regimens $(13,14)$.

The aims of the study were to determine the number of cases of mycobacterial disease, both TB and MOTT, in HIVpositive patients and in intravenous drug users (IVDU) treated in the period January 1986-December 1989 inclusive in the Department of Genito-Urinary Medicine, St. James' Hospital and Peamount Chest Hospital. Dublin. We considered the organisms involved, clinical presentation, radiological extent of disease, response to therapy and relapse rate.

This article is a reproduction of that published in: Respiratory Medicine, 1992, 86, pp. 491-494. Pagination may not match that of the original. 


\section{Methods}

We made a record of the chest X-ray appearances, bacteriological status, tuberculin status and response to treatment of all patients with mycobacterial disease known to be HIV-positive and also patients who were known to be IVDU. Tuberculin skin hypersensitivity is routinely tested using PPD-RT23 (Staten Serum Institute, Copenhagen) and the Mantoux technique.

All chest X-rays are read and classified in accordance with the recommendations of the National Tuberculous Association of the U.S.A. (15).

\section{Results}

On 1 January 1990 there were 910 known cases of HIV infection in the Republic of Ireland. One-hundred and twentyfour cases of AIDS had been diagnosed of whom 58 had died.

In the study period we treated 1000 cases of tuberculosis and 11 cases of MOTT. Eighteen patients were HIV-positive. We are aware of two further HIV-positive patients (both haemophiliacs) who were diagnosed as having TB but who were not admitted under our care and are not included in this study.

Of the 18 patients (15 males, three female) who were HIV-positive; 12 were IVDUs of whom one was also a prostitute and one a bisexual; six HIV-positive patients were homosexual/bisexual. Six patients with TB (three male, three female) were known IVDUs and HIV-negative.

Six of the HIV-positive patients had other features of CDC stage IV disease at the lime tuberculosis was diagnosed - PCP pneumonia and oral candidiasis (one); PCP pneumonia (one); oropharyngeal can-didiasis (three); recurrent bacterial pneumonia and anaemia (one). Three patients were receiving Zidovudine (AZT) at the time mycobacterial disease was diagnosed.

Seven HIV-positive patients and three HIV-negative patients were known to have received previous BCG vaccination. The chest X-ray appearances, tuberculin skin test results and organisms isolated are shown in Table 1. All strains of Mycobacterium tuberculosis isolated were fully sensitive. Extrapulmonary tuberculosis (genito-urinary TB, TB meningitis, cervival and axillary gland TB, ischiorectal abscess) occurred in ten HIV-positive patients and three HIVnegative patients.

Patients with TB were treated with standard therapy $(13,14)$ unless contraindicated by associated hepatic disease or drug intolerance. The patients with MOTT were treated with other drug combinations (Cycloserine $500 \mathrm{mg}$ b.d., Clofazamine $100 \mathrm{mg}$ t.d.s.,
Table 1 Radiological appearances, extent of disease, Mantoux status and Mycobacteria isolated in all patients

\begin{tabular}{lcc}
\hline & \multicolumn{2}{c}{ No. patients } \\
\cline { 2 - 3 } & $\begin{array}{c}\text { HIV- } \\
\text { positive } \\
(n=18)\end{array}$ & $\begin{array}{c}\text { HIV-negative/IVDU } \\
(n=6)\end{array}$ \\
\hline Chest X-ray & 12 & 0 \\
Atypical & 1 & 2 \\
Miliary & 1 & 0 \\
Pleural effusion & 0 & 1 \\
Minimal & 1 & 1 \\
Moderate & 2 & 2 \\
Advanced & 1 & 0 \\
Not available & 10 & 3 \\
Extrapulmonary TB & & \\
Mantoux result & 5 & 4 \\
Positive 1TU & 5 & \\
Positive 10TU & 2 & \\
Positive 100TU & 2 & 6 \\
Negative 100TU & 4 & 0 \\
Incomplete & & \\
Mycobacterium & 12 & \\
tuberculosis & 3 & \\
M.O.T.T. & 3 & \\
No mycobacteria & & \\
cultured & & \\
\hline
\end{tabular}

Rifabutin 300-600 mg daily, Clarithramycin 500mg b.d.)

Seven patients, (six HIV-positive and one HIV-negative IVDU) were lost to follow-up before completion of treatment. Four of these patients were positive on direct staining and culture for tuberculosis at the time they were lost to follow-up. Three HIV-positive patients died before completing treatment (one Mycobacterium kansasii, two non-mycobacterial causes). Two HIV patients, both with MOTT, have had no response to treatment. The remaining 12 patients (seven HIV-positive. live HIV-negative) have responded to treatment and completed their anti-tuberculous drug regimen.

Table 2 shows the time for patients to become consistently direct smear (S) and culture (C) negative for all patients. This is not significantly different from the conversion rates we have seen in trial protocol with standard antituberculous treatment (13). The six patients who were smear negative were unproductive of sputum. Three of these patients were positive on culture of broncho-alveolar lavage; one was positive on urine culture; one had a strongly positive ITM Mantoux with chest X-ray appearances consistent with tuberculosis; and one had therapy initiated in the U.K. where he had been positive on culture of pleural fluid for fully sensitive M.TB. This last patient 
Table 2 Time for sputum to become bacteriologically negative for M. tuberculosis

\begin{tabular}{ccccc}
\hline & \multicolumn{4}{c}{ No. patients } \\
\cline { 2 - 5 } $\begin{array}{c}\text { Duration of treatment } \\
\text { (weeks) }\end{array}$ & $\begin{array}{c}\text { HIV-positive } \\
(n=12)\end{array}$ & $\begin{array}{c}\text { HIV-negative/ } \\
\text { IVDU } \\
(n=6)\end{array}$ \\
\cline { 2 - 5 } & S- & C- & S- & C- \\
\hline 1 & 5 & 3 & 1 & 0 \\
4 & 7 & 8 & 4 & 4 \\
12 & 7 & 8 & 4 & 4 \\
26 & 7 & 8 & 5 & 5 \\
\hline
\end{tabular}

Four patients (three of whom were HIV-positive) were positive on direct staining and culture at the time they were lost to follow up. S-, Direct smear negative; C-, culture negative.

relapsed with fully sensitive Mycobacterium tuberculosis 3 months after completion of chemotherapy. He admitted ongoing drug abuse and poor compliance after 10-12 weeks of therapy. He has since become consistently negative on direct staining and on culture with supervised chemotherapy.

Statistical analysis using the $X^{2}$ technique comparing our experience between 1980 and 1985 (16) with 1986-1990 showed that there were statistically significantly more IVDUs treated for tuberculosis in the latter period $(P=<0.00001$; d.f. $\left.1, X^{2}=20.38\right)$. Analysis of patients admitted to Peamount in the latter period showed no significant geographical shift in referral pattern and we continued to treat $28-32 \%$ of all reported cases of tuberculosis in the Republic of Ireland. Consultation with the drug addiction treatment centres in Dublin have shown that all known IVDUs with TB attending the centres were treated by us.

\section{Discussion}

Twelve of the 24 patients described in this study were intravenous drug abusers. This shows a statistically highly significant increase in tuberculosis in IVDUs in Dublin since our previous study (16). Six of these patients were HIVpositive and these results suggest that the AIDS epidemic is contributing to an increased incidence of TB in the IVDU population in Dublin.

The numbers remain small but form a significant percentage of the total patients with AIDS and a significant workload because of the difficulty of managing patients, particularly those with manifestations of AIDS. Tuberculosis is also important in that it was the first indicator of Stage IV disease in 11 of 18 patients who were HIV-positive.

While advice with respect to safe sex and needle exchange programmes may help reduce the transmission of HI V. it will have no effect on the transmission of tuberculosis. One feature of the problem is that four patients (all IVDU) were lost to follow-up at a time when they were still positive on direct staining and culture. The organism will remain pathogenic (17) and because compliance in these patients is likely to be poor, they must be regarded as a serious ongoing source of transmission of tuberculosis.

While it has been suggested that patients with tuberculosis should perhaps be considered for HIV screening, experience in the Republic of Ireland to date suggests that this would not be appropriate or necessary at this time. It has been suggested $(11,18,19)$ that patients who are infected with HIV and have a positive skin test should be offered chemoprophylaxis or definitive treatment for tuberculosis to prevent them developing active disease. This may also help prevent the associated immunosuppression seen with tuberculosis (20) and perhaps prevent acceleration of progression to AIDS (2).

A difficulty in interpreting tuberculin skin test status in the Republic of Ireland is that the majority of young adults will have had BCG vaccination either as infants or at 12-14 years of age. In their study Selwyn et al. (6) could regard the Mantoux test as indicative of previous infection as routine BCG vaccination is not the practice in the United States. A further difficulty is deciding on the significance of skin anergy (negative 10 TU Mantoux) in patients infected with HIV as the negative test could indicate deteriorating T-cell function and progression towards AIDS. HIV-positive patients with chest symptoms or pyrexia of unknown origin and positive tuberculin skin tests must be assessed for active tuberculosis.

The duration of chemotherapy for patients with HIV AIDS for tuberculosis is not clear. These patients seem to respond equally well to therapy but there are problems with IVDUs particularly with compliance and other disease processes, particularly hepatitis B. We have had a policy of isoniazid prophylaxis indefinitely following completion of therapy because of possible reactivation and/or reinfection. Special procedures to ensure compliance with chemotherapy by IVDUs who have active tuberculosis are necessary and ideally they should receive fully supervised therapy.

Our study does not suggest that BCG gave useful protection against tuberculosis in these HIV-positive 
subjects but it was not designed to detect protection against tuberculosis from BCG. We have previously reported the protective effects of BCG on younger subjects in the Republic of Ireland $(21,22)$ but there is no reason to believe that this finding may be extrapolated to HIV-positive individuals.

\section{References}

1. Murray JF. The white plague: down and out or up and coming? Am Rev Respir Dis 1989; 140: 1788-1795.

2. Hopewell P. Tuberculosis and Human Immunodeficiency Virus infection. Sem Respir Infect 1989; 14: 111-122.

3. Tuberculosis and AIDS. Statement on AIDS and Tuberculosis by the Joint World Health Organisation/ International Union Against Tuberculosis and Lung Disease Working Group on HIV Infection and Tuberculosis. Bull IUATLD, 1989; 64: 8-11.

4. Caplin M, Grange JM, Morley S et al. Relationship between radiological classification and the serological and haematological features of untreated pulmonary tuberculosis in Indonesia. Tubercle 1989; 70: 103-1 13.

5. Beck JS, Potts RC, Kardjito T, Grange JM. T4 lymphopenia in patients with active pulmonary tuberculosis. din Exp Immunol 1985; 60: 49-54.

6. Selwyn PA, Hartel D, Lewis V et al. A prospective study of the risk of tuberculosis among intravenous drug users with Human Immunodeficiency Virus infection. New Engl J Med 1989; 320: 545-550.

7. Kelly P, Murphy A, Clancy L. Mycobactcrium tuberculosis hominis infection and AIDS. Ir Med J 1987; 80:271.

8. Collins C, Kelly P, Byrne C, Denham F, Clancy L. Is bovine, atypical or resistant tuberculosis a problem? Ir Med J 1987; 80:66-67.

9. Pitchenik AE, Cole C, Russell BW et al. Tuberculosis, atypical mycobacteriosis and AIDS among Haitian and non-Haitian patients in South Florida. Ann Int Med 1984; 101: 641-645.

10. Chaisson RE, Schechter GF, Theur CP et al. Tuberculosis in patients with the Acquired Immunodeficiency Syndrome. Clinical features, response to therapy and survival. Am Rev Respir Dis 1987; 136: 570-574.
11. Pitchenik AE, Robinson HA. The radiographic appearance of tuberculosis in patients with the Acquired Immunodeficiency Syndrome (AIDS) and pre-AIDS. $\mathrm{Am}$ Rev Respir Dis 1985; 131: 393-396.

12. Sunderam G, MacDonald RJ, Moniatis $\mathrm{T}$ et al Tuberculosis as a manifestation of AIDS. $J \mathrm{Am} \mathrm{Med}$ Assoc 1986; 256: 362-366.

13. Howell F, O'Laoide R, Kelly P, Power J, Clancy L Short Course chemotherapy for pulmonary tuberculosis: a randomised controlled trial of a six month versus a nine month oral regimen. Ir Med J 1989; 82: 11-13.

14. Antituberculosis regimens of chemotherapy. Recommendations from the Committee on Treatment of the International Union Against Tuberculosis and Lung Disease. Bull IUA TLD 1988; 63: 60-64.

15. National Tuberculosis Association of the U.S.A. Diagnostic standrds and Classification of Tuberculosis New York: National Tuberculosis Association of the U.S.A., 1961.

16. Howell F, Kelly P, Clancy L. Pulmonary tuberculosis in the Republic of Ireland: an epidemiological profile from a single unit. Respir Med 1990; 84: 111-117.

17. Clancy L, Kelly P, O'Reilly L, Byrne C, Costello E. The pathogenicity of Mycobacterium Tuberculosis during chemotherapy. Eur Respir J 1990; 3: 399-402.

18. Pitchenik AE, Burr J, Cole CH. Tuberculin testing for persons with positive serologic studies for HTLV-III. New Engl J Med 1986; 314: 447.

19. Van Deutekom H. Chemoprophylaxis and chemotherapy of tuberculosis in HIV infected patients. Bull IUATLD 1990; 65: 84-85.

20. Katz P, Goldstein RA, Fauci AS. Immunoregulation infection caused by $M$. tuberculosis; the presence of suppressor monocytes and the alteration of subpopulations of T lymphyocytes. J Infect Dis 1979; 140: $12-21$.

21. Shannon A, Kelly P, Lucey M, Cooney M, Corcoran Clancy L. Isoniazid resistent tuberculosis in a school break: the protective effect of BCG. Eur Respir J 1991; 4: 778-782.

22. Clancy L, Shannon A, Lucey M et al. Evaluation of neonatal BCG vaccination in Ireland. Am Rev Respir Dis 1990; 141: A894 (1990 World Conference on Lung Health). 